\title{
PADE APPROXIMANTS AND THE ANHARMONIC OSCILLATOR
}

\author{
J. J. LOEFFEL * \\ linivesity of Lausanne. Switzerland \\ A. MARTIN \\ CERN, Goncra. Swilzerland \\ and \\ B. SIMON and A.S. WIGHTMAN \\ Departmont of Mallinatics. Princeton linitersity. Princelon. N.J.. I'SA
}

Received 27 vovember 1969

\begin{abstract}
The diagomal pade approximants of the perturbition series for the cigenvalues of the anharmonic oscillator (a $\beta \kappa^{t}$ perturbition of $\beta^{2} \cdot \kappa^{2}$ ) converge to the eigenvalues.
\end{abstract}

Recently there has been considerable interest in applyingr the method of Padé approximants $\{1\}$ to strong interaction physics $|2|$. This interest is based on the assumption that the diagonal Partés based on the Feynman series for the partial wave scattering amp!itude converge to the "correct answer". We report here a study of the Parté approximants for the energy levels. $E_{n}(\beta)$. of the anharmonic oscillator whose Ha niltonian is $p^{2}+\kappa^{2}+i 3 \kappa^{4}$. Our main resilt is that the diagonal Pade's !jased on the Rayleigh-Schrödinger series for an $\kappa^{\frac{1}{2}}$ perturbation of $p^{2}+\kappa^{2}$ converge for any eigenvalue and that the limit is the actual eigrenvalue.

We feel that this result is of some interest both in itself. and in relation to the work of Bessis et al. and Copley and Masson. The Hamiltonian $p^{2}+\kappa^{2}+\beta \kappa^{4}$ is closely analogous to a field theory with the Hamiltonian density :-in 2 + $\left.+:(\nabla \odot)^{2}:+m^{2}: \phi^{2}:+\beta: \leftrightarrow\right)^{4}:$. The analogy is strengthened by the fact that the perturbation series for the Green's function diverge in both cases. For the anharmonic oscillator it has been proved and for the field theory it is hoped that the series is asymptotic to the actual Green's function. What we prove here is that for the c'ige'malues of the anharmonic oscillator. the Padé approximants formed from the divergent Rayleigh-Schrödinger perturbation series converge to the right answer.

We first recall that the Pade approximants

\footnotetext{
- Cuder contract of C.1.C.P.
}

associated with a formal power series. $\sum a_{n} z^{n}$. are defined as follows: $f^{|. M . M|}$ is that unique rational function of degree $M$ in the numerator and $N$ in the denominator satisfying

$f^{|N \cdot M|}(z)-\sum_{0}^{M+N} a_{n} z^{n}=\mathrm{O}\left(z^{N+M+1}\right)$

Our proof of convergence will depend on anal$y$ tic properties recently established for the anharmonic oscillator energy levels as functions of the coupling constant ${ }^{\dagger}\{4,5\}$. Explicitly, we use:

(a) $E_{n}(\beta$ ) has an analytic continuation to a cut plane. cul along the negative real axis $\$$.

We return to a proof of this fact. which is the heart of the argument. near the conclusion of the note.

(b) $\operatorname{Im} E_{i:}(3) \quad 0$ if $\operatorname{Im} \beta \quad 0$.

This follows from the simple observation $\operatorname{Im} E_{n}(\beta)=\operatorname{Im} \beta\left\langle. x^{4}\right\rangle$.

(c) The Rayleigh-Schrödinger series is asymptotic to $E_{n}(\beta)$ as $\beta \rightarrow 0$. uniformly in $\arg \beta ; \pi$.

For $\beta 0$. this follows from results of Kato $[7 \mid$. For arbitrary $\beta$. it can be proved directly

t The carliest studies of analyticity used a non-rigorous WKB related approximation [3]. In the field theory case. there are no exact theories whose analylic properties can be similarly analyod. However. one is very cluse 10 il $10^{+}$, 2 theory for which the Pade approximants might convergolij].

F This is a non-trivial statement since $E_{n}(\beta)$ has infinitely malny hanch points near $\beta$ o $[1]$. "lhey happen to be on the second sheet. 
using Hilbert space argunents [4] or from Kato's results and the analytic and positivity properties (a). (b) $[5]$.

(d) For $\beta$ large and fixed $n,\left|E_{n}(\beta)\right| C|\beta| 1 / 3 *$.

Consider the Hamiltonian $p^{2}+\alpha k^{2}+\beta \kappa^{4}(\alpha$ real. $\beta$ 0) with eigenvalues $E_{n}(\alpha . \beta)$. As Symanzik has pointed out $[8]$. since the scaling $p \rightarrow \beta^{1 / i} p$; $\kappa \cdots \cdot \beta^{-1 ;} \kappa$ is unitarily implementable. $E_{n}(1, \beta)=$ $=\beta^{1 / 3} E_{n}\left(\beta^{-213}, 1\right)$ for $\beta$ real. By analytic continuation. this holds in the entire cut $\beta$ plane. Since $E_{n}(\alpha .1)$ is analytic at $\alpha=0$. the bound follows with any $C \quad E_{n}(0.1)$.

(e) Fix $n$. If $a_{m}$ are the Rayleigh-Schrödinger coefficients for $E_{n}(\beta)$. then $a_{m} \therefore C D^{m} m^{m}$.

This follows from the usual recursive relations for the $a_{m}$ by an inductive argument [4].

Now one proves that any diagonal Fadé sequence. $f^{[N . N \cdot j \mid}(\beta)$ ( $j$ fixed), for an eigenvalue. $E(\beta)$. converges uniformly on compacts of the cut plane. From (a). (b). (c) and (d), it follows that

$a_{n}=(-1)^{n+1} \int_{0}^{\infty} \gamma^{n} \mathrm{~d} \rho(\gamma)$ for $n \because 1$

where

$\mathrm{d} \rho(\gamma)=\lim _{\epsilon \rightarrow 0^{+}}[\pi \gamma]^{-1} \operatorname{Im} E\left(-\gamma^{-1}+\mathrm{i} \epsilon\right) \mathrm{d} \gamma$

From (b). we conclude that $d \rho(\gamma)$ is a positive measure so that $\left(-a_{n}\right)$ defines a series of Stieltjes. It thus follows from general theorems on Pade approximants $[1]$. that $f^{[N . N \cdot j]}$ converges for any fixed $j \dagger$. say to $f_{j}(\beta)$. Each $f_{j}$ obeys (a). (b). (c) and thus both (2) and

$$
\mathrm{d} \rho_{j}(\gamma)=\lim _{\epsilon \rightarrow 0^{+}}(\bar{i} \gamma)^{-1} \operatorname{Im} f_{j}(-\gamma-1+i \epsilon) \mathrm{d} \gamma
$$

solve the moment problem for the $\left(a_{n}\right)$. i.e.. obey (1). By (e). $\Sigma \cdot\left|a_{n}\right|^{-1 /(2 n+1)}=\infty$ so. by a theorem of Carleman $[1] . n^{n} \rho=\rho_{j}$. Thus $f_{j}-E$ is entire and has a zero asymptotic series. i.e.. $f_{j}-E=0$. This completes the proof.

We have made numerical calculations for the ground state to check the rate of convergence of the Padé approximants. In table 1 . we list

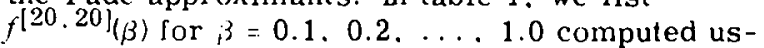
ing the Rayleigh-Schrödinger coefficients found by Bender and Wu $|3|$. We compare $f^{20.20 \mid}$ with

" I'sing b) alone. one (an plore $E_{n}(3)|C| \beta \mid$. This would imply (l) for " 2 which would suffice for our results.

† In rof. 1. this is only proved for $j \cdot 0$. when eq. (1) holds! However. $(-E(\beta))^{-1}$ obers (it)-(d) with the inverse power series so $\left.\left(-E^{-1}\right) \mid N . . V \cdot j\right]-E[N \cdot j . N \mid$ converges. One of us (B.S.) would like to thank Professor D. Masson for a discussion of this point.
Tabble 1

Comparison of Padé with rigorous bounds.

\begin{tabular}{|c|c|c|c|}
\hline i3 & Upler boundata & Lomer bound (b) & $f^{120.201}(c)$ \\
\hline 0.1 & 1.065286 & 1.065285 & $1.0652855005+3$ \\
\hline 0.2 & 1.118293 & 1.118 .292 & $1.1182926 .5+3(57)$ \\
\hline 0.3 & 1.16 .4055 & 1.16 .10 .11 & $1.1640+7156(23) 4)$ \\
\hline 0.4 & 1.20 .48 .18 & 1.204791 & 1.20481031106033 \\
\hline $0 . \bar{j}$ & $1.2+1957$ & $1.2+1 \times 11$ & $1.2418539(4 \times 135)$ \\
\hline 0.6 & 1.276195 & 1.275909 & $1.275983(105974)$ \\
\hline 0.7 & $1.30 \times 110$ & $1.307324^{1.1}$ & $1.3077+71246.301)$ \\
\hline 0.8 & 1.338096 & 1.333739 & $1.33754(1726.579)$ \\
\hline 0.9 & 1.266442 & $1.3643 .49(1)$ & $1.36566(23998911)$ \\
\hline 1.0 & 1.393371 & 1.392131 & $1.392331374 \times 1 \times 611$ \\
\hline
\end{tabular}

(i) From Bat ley-Fox $|12|$. table 1. A Rayle igh-Rit\% method was used on the first five eren parity levels. (b) From IReid|12|. table 3 axcept as noted by 1.1 which are taken from Bayley-Fox|121.

(e) We have thrown out the last three digits from at double precision answer assuming them insignificant because of round-offerror. The figures in parentheses

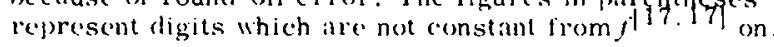

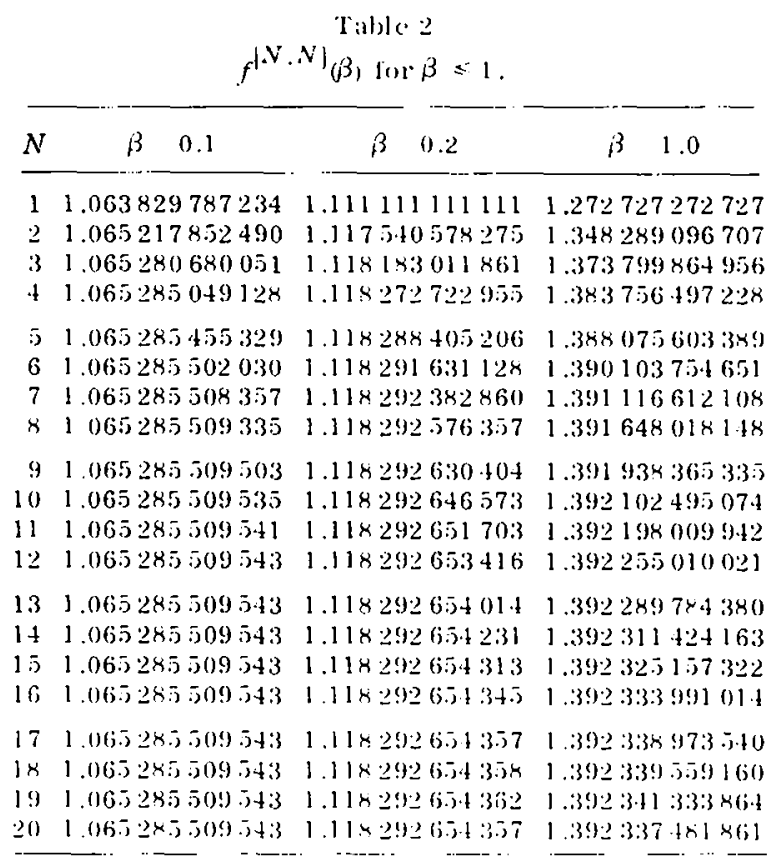

rigorous upper and lower bounds as computed by Bazley-Fox and Reid $[9 \mid \$$. We note for comparison that the sum of the first 41 terms of the Rayleigh-Schrödinger series is of order $10^{26}$

$\mp$ Notice that we give this lower hound only as a check of the numerical calculations. Indeed $|W N . N|$. for positive $\beta$ is itself necessarily a lower bound of $E(\beta)$. 
even for $\beta=0.1$. In table 2 , we show the rate of convergence of $f^{(N . N)}(\beta)$. This get worse as $\beta$ increases which is to be expected since $f^{[N, N]}(\beta) \sim$ some constant $C_{N}$ as $\beta \rightarrow \infty$ while $E(\beta) \sim C \beta^{1 / 3}$ as $\beta \rightarrow \infty$.

Let us return to the proof of (a). the cut plane analyticity for $E_{\eta l}(\beta)$. The absence of poles and non ramified isolated essential singularities for $\operatorname{Im} \beta \neq 0$ is a direct consequence of the Herglot $z$ property (b) $[4,5]$. When $\beta$ is real and positive. analyticity is a consequence of the Kato-Rellich theorems on regular perturbations.

To eliminate natural boundaries and branch points a more detailed study is needed $[5]$. The best characterization of an energy level for real $\alpha$ and $\beta \quad 0$ is the number of zeros of its wave function in $x$ space. It turns out that this notion can be generalized to complex $\alpha$ and $\beta$. Let us start from the wave equation

$H \psi=\left(-\frac{\mathrm{d}^{2}}{\mathrm{~d} x^{2}}+\alpha x^{2}+x^{4}\right) \psi=E(\alpha, 1) \psi(x, \alpha, E)$

with the boundary condition

$\psi \sim \frac{1}{x} \exp -\frac{1}{3}, 3$ for $x \rightarrow+\infty$

The energy levels are given implicitly by

$$
\psi(x=0, \alpha, E)=0 \text { for odd levels }
$$

$\frac{\partial}{\partial x} \psi(x=0 . \alpha . E)=0$ for even levels

where $\psi(x=0, \alpha, E)$ is entire in $\alpha$ and $E$. Around a point $\alpha_{0} E_{0}$. where $E_{0}$ is finite. the energy is an analytic function of a fractional power of $\alpha-\alpha_{0}$.

What we can prove by integrating $\psi^{*}(z)[H-E] \times$ $\times \psi(z)$ along rays in the complex $z$ plane is the following: for $|\arg \alpha| \frac{2}{3} \pi-\epsilon, \epsilon$ arbitrarily small. $|\psi|$ is strictly positive for

$\frac{1}{1 i} \pi-\epsilon^{\prime} \quad \arg z \quad \frac{1}{6} \pi \quad$ and $-\frac{1}{6} \pi \cdots \arg z-\frac{1}{6} \pi+\epsilon^{\prime}$ and for $|z|$ large if $|\arg z| \frac{1}{6} \pi$. Therefore if we vary $\alpha$ continuously and hence $E$ continuously (if it does nol go (hrough infinity' the number of zeros of the wave functions in the sector $|\arg z|$ $\therefore \frac{1}{5} \pi$ cannot vary. That $E$ will remain bounded during this continuous motion in the $\alpha$ plane is established as follows: when we start. with $\alpha$ on the real axis. we have a finite number of zeros $n$ in this sector, all of which are real. Now integrating the wave equation from the origin in the Volterra form we can prove that $E$ cannot get too large for complex $\alpha$ because if it did the "free" solution $\sin (\sqrt{E} z)$ or $\cos (\sqrt{E} z)$ would dominate for finite $|z|$ and. applying the Rouché theorem to a suitable finite region inside $|\arg z|$

$\frac{1}{n} \pi$ we would get a number of zeros Iarger than $n$. which would be a contradiction*.

Since $E$ remains bounded, the only possible singularities of $E(\alpha)$ are branch points. However. if we turn around such a branch point and come back to the real axis we fall back on a real wave function with the same number of zeros $z$ as the one we started from. Therefore there cannot be any branch point for $|\arg \alpha| \frac{3}{3} \pi$. If we return through scaling to the variable $\beta$ we find that all energy levels $E_{n}(\beta)$ are analytic in a cut plane.

Finally let us discuss the extension to $\kappa^{2 m}$ perturbations and several dimensions. For $\kappa^{2 m}$ perturbations. there are indications that $a_{n} \sim$ $\sim c D^{n} n^{(m-1) n}$ so that Carleman's criterion $\sum\left|a_{n}\right|^{-1 /(2 n+1)}=\infty$ breaks down at $\kappa^{8}$. Since Carleman's criterions is sufficient but not necessary, our proof that $f_{j}=E$ breaks down but the equality mav still hold. A numerical analysis of this $\kappa^{8}$ problem is in progress $[10]$. Similarly for several dimensional coupled anharmonic oscillators, one part of the proof breaks down: for the proof that $E_{n}(\beta)$ has no branch points in the cut plane depends on keeping track of zeros. a more complicated affair in several variables.

It is a pleasure to thank A. Dicke. H. Epstein. V. Glaser. D. Masson, E. Stein and K. Symanzik for very valuable comments. Two of us (A. M. and B.S.) are grateful to N. N. Khuri for arranging a meeting which stimulated this work.

* We hope to find an a rgument which does not make explicit use of the wave equation to show that $E$ remains bounded. but the matter is not yet completely clear. It would obviously be better for it could be generalized to more degrees of freedom.

\section{References}

1. G. A. Baker. Adv. Theoret. Phys. 1 (1965) 1.

2. D. Bess is and M. Pusterla. Nuovo Cimento $54 \mathrm{~A}$ (1968) 243:

$J$ L. Basdevant, D. Bessis and J.Zinn-Justin. Nuovo Cimento 60A (1969) 185 :

D. Bessis. S. Gratti. V.Grecchi and G. Turchetti. Phys. Letters 28B (1969) 8:

L. Copley and D. Masson. Phys. Rev. 164 (1967) 2059.

3. C. Bender and T. T. Wu. Phys. Rer. Letters 21 (1968) 406: Phys. Rev.. to be published.

t. B. Simon. Coupling constant analytieity for the anharmonic oscillator. Princeton Preprint (September 1969). Submitted to Ann. Phys. (N.Y.).

5. J. J. Loeffel and A. Martin. in preparation.

6. J. Glimm and A Jaffé. Phys. Rev. 176 (1968) 1945: J. Glimm and A.Jaffe. N.Y. Lniversity Preprints: $J$. Cannon and A. Jaffé. in preparation.

7. T. Kato. Progr. Theor. Phis. 1 (1949) $514: 5$ (1950) 95: 207.

8. K. Symanzik. private communication.

9. N. Bazley and D. Fox. Phrs. Rev. $12+$ (1961) 483 : C Reid. J. Chem. Phys. 43 (1965) S186.

10. T. Karr private communication. 